\title{
THE STRICT TOPOLOGY FOR DOUBLE CENTRALIZER ALGEBRAS $\left({ }^{1}\right)$
}

\author{
BY \\ DONALD CURTIS TAYLOR
}

\begin{abstract}
Sufficient conditions are given for a double centralizer algebra under the strict topology to be a Mackey space.
\end{abstract}

0 . Introduction. Let $C(S)$ be the $B^{*}$-algebra of all bounded complex valued continuous functions on a locally compact Hausdorff space $S$; let $C_{0}(S)$ be the algebra of all functions in $C(S)$ that vanish at infinity, and let $C(S)_{\beta}$ denote $C(S)$ under the $\beta$ or strict topology. In 1958, R. C. Buck [3] proved that the strict dual of $C(S)$ under the strong topology is isometrically isomorphic to the norm dual of $C_{0}(S)$ and then raised the following question: Is it in fact true that the strict topology $\beta$ coincides with the Mackey topology? In 1967, J. B. Conway [6] answered this question for the most part. He showed that if $S$ is paracompact, then indeed the strict topology is the Mackey topology and he also gave examples of locally compact spaces $S$ where the strict topology for $C(S)$ is not the Mackey topology.

More recently, R. C. Busby [4] in his study of double centralizers of $B^{*}$-algebras introduced a generalized notion of the strict topology. Specifically, if $A$ is a $B^{*}$ algebra and $M(A)$ is its double centralizer algebra, then the strict topology $\beta$ for $M(A)$ is defined to be that locally convex topology generated by the seminorms $\left(\lambda_{a}\right)_{a \in A}$ and $\left(\rho_{a}\right)_{a \in A}$, where $\lambda_{a}(x)=\|a x\|$ and $\rho_{a}(x)=\|x a\|$, and we let $M(A)_{\beta}$ denote $M(A)$ under the strict topology. Although Busby investigated some of the properties of the strict topology in this setting, no mention was made of the strict dual of $M(A)$. Thus, the questions under consideration are the following: (1) Is the strict dual of $M(A)$ under the strong topology a Banach space that is isometrically isomorphic to the norm dual of $A$ ? (2) What are some sufficient conditions for the strict topology for $M(A)$ to be the Mackey topology? The answer to question (1) is yes and to answer question (2) we prove the following two theorems:

TheOREM I. Let $\left\{A_{\lambda}: \lambda \in \Lambda\right\}$ be a family of $B^{*}$-algebras and let $A=\left(\sum A_{\lambda}\right)_{0}$. Then $M(A)_{\beta}$ is a Mackey space if, and only if, for each $\lambda \in \Lambda, M\left(A_{\lambda}\right)_{\beta}$ is a Mackey space.

Received by the editors October 14, 1969.

AMS Subject Classifications. Primary 4650, 4665; Secondary 4601.

Key Words and Phrases. Double centralizer algebras, $B^{*}$-algebras, $C^{*}$-algebras, strict topology, Mackey topology.

(1) This research was supported in part by the National Science Foundation, under contract No. GP-15736. 
TheOREM II. Let $A$ be a $B^{*}$-algebra and suppose one of the following conditions holds:

(1) $M(A)$ is isometrically *-isomorphic to the bidual of $A$.

(2) $A$ has a countable approximate identity.

Then $M(A)_{\beta}$ is a Mackey space.

If $S$ is a locally compact paracompact Hausdorff space, then by [2, p. 107] $S$ can be expressed as the union of a collection $\left\{Y_{\lambda}: \lambda \in \Lambda\right\}$ of pairwise disjoint open and closed $\sigma$-compact subsets of $S$. For each $\lambda \in \Lambda$ set $A_{\lambda}=C_{0}\left(Y_{\lambda}\right)$ and observe that $A_{\lambda}$ has a countable approximate identity. Since $A$ and $M(A)$ are isometrically *-isomorphic to $C_{0}(S)$ and $C(S)$ respectively, where $A=\left(\sum A_{\lambda}\right)_{0}$, it follows that Theorem II, together with Theorem I, generalizes Conway's result [6, Theorem 2.6, p. 478] as well as a result of LeCam [11, Proposition 3, p. 220].

Furthermore Theorem II, together with the fact that the strict dual of $M(A)$ under the strong topology is isometrically isomorphic to the norm dual of $A$, gives for a special case a characterization of the Mackey topology of $W^{*}$-algebras (see [1]).

1. Notation and preliminaries. Let $A$ be a $B^{*}$-algebra. By a double centralizer on $A$, we mean a pair $(R, S)$ of functions from $A$ to $A$ such that $a R(b)=S(a) b$ for $a, b$ in $A$, and we will denote the set of all double centralizers on $A$ by $M(A)$. If $(R$, $S) \in M(A)$, then $R$ and $S$ are continuous linear operators on $A$ and $\|R\|=\|S\|$, so $M(A)$ under the usual operations of addition and multiplication is a Banach algebra, where $\|(R, S)\|=\|R\|$. Furthermore, if we define $(R, S)^{*}=\left(S^{*}, R^{*}\right)$, where $R^{*}(a)=\left(R\left(a^{*}\right)\right)^{*}$ and $S^{*}(a)=\left(S\left(a^{*}\right)\right)^{*}$ for all $a \in A$, then $(R, S)^{*} \in M(A)$ and this implies that $M(A)$ is a $B^{*}$-algebra. If we define a map $\mu_{0}: A \rightarrow M(A)$ by the formula $\mu_{0}(a)=\left(L_{a}, R_{a}\right)$, where $L_{a}(b)=a b$ and $R_{a}(b)=b a$ for all $b \in A$, then $\mu_{0}$ is an isometric *-isomorphism from $A$ into $M(A)$ and $\mu_{0}(A)$ is a closed two sided ideal in $M(A)$. Hence throughout this paper we will view $A$ as a closed two sided ideal in $M(A)$. If $A$ is commutative, then $M(A)$ is isometrically $*$-isomorphic to the algebra of multipliers as studied by Wang [17]. If $\left\{A_{\lambda}\right\}$ is a family of $B^{*}$ algebras, then $\sum A_{\lambda}$ and $\left(\sum A_{\lambda}\right)_{0}$ are defined as in [12]. It is clear that $\sum A_{\lambda}$ and $\left(\sum A_{\lambda}\right)_{0}$ are $B^{*}$-algebras. For a more detailed account of the theory of double centralizers on a $B^{*}$-algebra, we refer the reader to [4], and for definitions and concepts in general, we refer the reader to [10] and [12].

2. The dual of $M(A)_{\beta}$. In this section we prove that the strict dual of $M(A)$ under the strong topology is isometrically isomorphic to the norm dual of $A$ and furthermore, we characterize the $\beta$-equicontinuous subsets of the strict dual of $M(A)$.

Theorem 2.1. Let $A$ be $a B^{*}$-algebra and let $A^{*}$ denote the dual of $A$. Then $A^{*}=\left\{a \cdot f: a \in A\right.$ and $\left.f \in A^{*}\right\}=\left\{f \cdot a: a \in A\right.$ and $\left.f \in A^{*}\right\}$, where $a \cdot f(b)=f(b a)$ and $f \cdot a(b)=f(a b)$ for all $b \in A$. 
Proof. Let $f$ be a positive linear functional in $A^{*}$. By virtue of [12, Theorem 4.5.14, p. 219] $f$ is representable; that is, there exists a Hilbert space $H$, a continuous *-representation $a \rightarrow T_{a}$ of $A$ on $H$, and a topologically cyclic vector $h_{0}$ in $H$ such that $f(a)=\left(T_{a} h_{0}, h_{0}\right)$ for all $a \in A$. Let $\left\{e_{\lambda}\right\}$ be an approximate identity for $A$. Since $h_{0}=\lim T_{a_{n}} h_{0}$ for some sequence $\left\{a_{n}\right\}$ of elements in $A$, we can easily show that $\lim T_{e_{\lambda}} h_{0}=h_{0}$. Due to the fact that $H$ is an $A$-module in the sense of [9, Definition 2.1, p. 147], we have by the Cohen-Hewitt factorization theorem [9, Theorem 2.5, p. 151] that $h_{0}=T_{a} h_{1}$ for some $a \in A$ and $h_{1} \in H$. Define $g$ on $A$ by the formula $g(b)=\left(T_{b} h_{1}, h_{1}\right)$ for each $b \in A$ and note that $g \in A^{*}$ and $f=a \cdot g \cdot a^{*}$.

Now assume that $f$ is any element of $A^{*}$. Since $f$ can be expressed as a finite linear combination of positive functionals on $A$ [14, Theorem 1, p. 439], we see that $\lim e_{\lambda} \cdot f=\lim f \cdot e_{\lambda}=f$. Hence, by [9, Theorem 2.5, p. 151], there exist elements $a$ and $b$ in $A$ and linear functionals $g_{1}$ and $g_{2}$ in $A^{*}$ such that $f=a \cdot g_{1}=g_{2} \cdot b$ and our proof is complete.

Corollary 2.2. If $A$ is $a B^{*}$-algebra, then $M(A)_{\beta}^{*}=\left\{a \cdot f: a \in A\right.$ and $\left.f \in M(A)^{*}\right\}$ $=\left\{f \cdot a: a \in A\right.$ and $\left.f \in M(A)^{*}\right\}$, where $a \cdot f(x)=f(x a)$ and $f \cdot a(x)=f(a x)$ for all $x \in M(A)$.

Proof. Due to the fact that the strict topology is weaker than the norm topology, we have that $M(A)_{\beta}^{*} \subset M(A)^{*}$. Now let $f \in M(A)_{\beta}^{*}$ and let $\phi f$ denote the restriction of $f$ to $A$. By Theorem 2.1 there exists an $a \in A$ and a $g \in A^{*}$ such that $\phi f=a \cdot g$. By the Hahn-Banach theorem there exists an $h \in M(A)^{*}$ such that $g=\phi h$. Now let $\left\{e_{\lambda}\right\}$ be an approximate identity for $A$ and let $x \in M(A)$. Since $e_{\lambda} x+x e_{\lambda}-e_{\lambda} x e_{\lambda}$ converges to $x$ in the strict topology and $A$ is a closed two sided ideal in $M(A)$, we have that

$$
\begin{aligned}
f(x) & =\lim f\left(e_{\lambda} x+x e_{\lambda}-e_{\lambda} x e_{\lambda}\right)=\lim a \cdot g\left(e_{\lambda} x+x e_{\lambda}-e_{\lambda} x e_{\lambda}\right) \\
& =g(x a)=h(x a)=a \cdot h(x) .
\end{aligned}
$$

Hence $f=a \cdot h$ and similarly there is a $b \in A$ and an $h_{1} \in M(A)^{*}$ such that $f=h_{1} \cdot b$. Since it is easy to show that $a \cdot f$ and $f \cdot a$ are strictly continuous for each $a \in A$ and $f \in M(A)^{*}$, our proof is complete.

The strong topology for $M(A)_{\beta}^{*}$ is defined to be the topology of uniform convergence on the $\beta$-bounded subsets of $M(A)_{\beta}$.

Corollary 2.3. If $A$ is a $B^{*}$-algebra, then $M(A)_{\beta}^{*}$ under the strong topology is a Banach space that is isometrically isomorphic to $A^{*}$.

Proof. By virtue of the uniform boundedness principle, it is straightforward to show that the $\beta$-bounded subsets of $M(A)$ are norm bounded. Therefore, the strong topology for $M(A)_{\beta}^{*}$ is the usual topology generated by the norm of $M(A)^{*}$. Since $A$ is strictly dense in $M(A)_{\beta}$, we have by Theorem 2.1 and Corollary 2.2 that the restriction map $\phi$ is an isomorphism of $M(A)_{B}^{*}$ onto $A^{*}$. Therefore, to complete the proof we need to show that $\phi$ is an isometry. But this follows from the fact 
that $f(x)=\lim f\left(x e_{\lambda}\right)$ for each $f \in M(A)_{\beta}^{*}$ and $x \in M(A)$, where $\left\{e_{\lambda}\right\}$ is an approximate identity for $A$.

Lemma 2.4. Let $A$ be a $B^{*}$-algebra and let $\left\{d_{n}\right\}$ be a sequence of elements of $A$, $\left\|d_{n}\right\|<1$, that converges to zero. Then there exist sequences $\left\{b_{n}\right\}$ and $\left\{c_{n}\right\}$ of elements of $A$ and a hermitian element $a$ of $A,\|a\| \leqq 1$, such that

(1) $d_{n}=a b_{n}=c_{n} a$;

(2) $\left\|d_{n}\right\| \geqq \max \left\{\left\|b_{n}\right\|^{2},\left\|c_{n}\right\|^{2}\right\}$.

Proof. Let $A_{1}$ be the $B^{*}$-algebra obtained by adjoining the identity, let $\left\{e_{\lambda}\right\}$ be an approximate identity for $A$ consisting of hermitian elements, and let $Z=\left\{x \in A: x=d_{n}, x=d_{n}^{*}, x=\left(d_{n} d_{n}^{*}\right)^{1 / 4}\right.$, or $\left.x=\left(d_{n}^{*} d_{n}\right)^{1 / 4}\right\}$. Since $e_{\lambda} x \rightarrow x$ uniformly on $Z$, we may define by induction a sequence $\left\{e_{\lambda_{k}}\right\}$ of elements in the unit ball of $A$ such that $\left\|x-e_{\lambda_{n}} x\right\|<\delta / 8^{n+1}, x \in Z$, and $\left\|e_{\lambda_{k}}-e_{\lambda_{n+1}} e_{\lambda_{k}}\right\|<\delta / 32^{n+1}, k=1,2, \ldots, n$, where $\delta=\min \left\{1-\left\|d_{n}\right\|^{1 / 2}: n=1,2,3, \ldots\right\}$. Now set

$$
a_{n}=\sum_{k=1}^{n} \nu(1-\nu)^{k-1} e_{\lambda_{k}}+(1-v)^{n}, \quad \text { where } 0<\nu<1 / 4 \text {. }
$$

It follows, as in the proof of [16, Theorem 2.1], that $a_{n}^{-1}$ exists, $\left\|a_{n}^{-1}\right\| \leqq 4^{n}$, and $a_{n+1}^{-1}-a_{n}^{-1}=r\left(1-e_{\lambda_{n+1}}\right)+s$, where $\|r\| \leqq 4^{n}$ and $\|s\| \leqq \delta / 2^{n+2}$. These facts together with the fact that $a_{n}^{-1}$ is hermitian gives us, as in the proof of [16, Theorem 2.1], that $\lim a_{n}^{-1} x$ and $\lim x a_{n}^{-1}$ exist for each $x \in Z$ and that $\left\|\lim a_{n}^{-1} x\right\| \leqq\|x\|+\delta$. So, by setting $b_{n}=\lim _{p \rightarrow \infty} a_{p}^{-1} d_{n}, c_{n}=\lim _{p \rightarrow \infty} d_{n} a_{p}^{-1}$, and $a=\lim a_{p}$, we see that (1) holds. We now wish to show that (2) holds. But

$$
\begin{aligned}
\left\|b_{n}\right\|^{2} & =\left\|b_{n} b_{n}^{*}\right\|=\lim _{p \rightarrow \infty}\left\|a_{p}^{-1} d_{n} d_{n}^{*} a_{p}^{-1}\right\|=\lim _{p \rightarrow \infty}\left\|a_{p}^{-1}\left(d_{n} d_{n}^{*}\right)^{1 / 4}\left(d_{n} d_{n}^{*}\right)^{1 / 2}\left(d_{n} d_{n}^{*}\right)^{1 / 4} a_{p}^{-1}\right\| \\
& \leqq\left(\left\|d_{n} d_{n}^{*}\right\|^{1 / 4}+\delta\right)^{2}\left\|d_{n}\right\| \leqq\left\|d_{n}\right\| .
\end{aligned}
$$

Similarly $\left\|c_{n}\right\|^{2} \leqq\left\|d_{n}\right\|$ and (2) holds.

LemMA 2.5. Let $A$ be a $B^{*}$-algebra. The collection of all sets

$$
V_{a}=\{x \in M(A):\|a x\| \leqq 1 \text { and }\|x a\| \leqq 1\}
$$

for $a \in A$ is a base at 0 in $M(A)$ for the strict topology.

Proof. The proof follows from a straightforward application of Lemma 2.4.

THEOREM 2.6. Let $A$ be a $B^{*}$-algebra and let $\left\{e_{\lambda}: \lambda \in \Lambda\right\}$ be an approximate identity for $A$. If $H$ is a subset of $M(A)_{\beta}^{*}$, then the following statements are equivalent:

(1) $H$ is $\beta$-equicontinuous.

(2) $H$ is uniformly bounded and $e_{\lambda} \cdot f+f \cdot e_{\lambda}-e_{\lambda} \cdot f \cdot e_{\lambda} \rightarrow f$ uniformly on $H$, where $e_{\lambda} \cdot f(x)=f\left(x e_{\lambda}\right)$ and $f \cdot e_{\lambda}(x)=f\left(e_{\lambda} x\right)$ for all $x \in M(A)$.

Proof. Assume (1) holds. Then $H$ is contained in the polar of some basic neighborhood $V_{a}=\{x \in M(A):\|a x\| \leqq 1$ and $\|x a\| \leqq 1\}$ of 0 . Since the $\beta$-topology is weaker than the norm topology, it follows that $H$ is uniformly bounded. Now for 
each $x \in M(A)$ and $\varepsilon>0$ the element $x /(\|a x\|+\|x a\|+\varepsilon)$ belongs to $V_{a}$. So for $f \in H$

$$
\begin{aligned}
|f(x)| & =|(\|a x\|+\|x a\|+\varepsilon) f(x /(\|a x\|+\|x a\|+\varepsilon))| \\
& <\|a x\|+\|x a\|+\varepsilon .
\end{aligned}
$$

Since $\varepsilon$ was picked arbitrarily, it follows that $|f(x)| \leqq\|a x\|+\|x a\|$. Hence

$$
\begin{aligned}
\left|\left(f-e_{\lambda} \cdot f-f \cdot e_{\lambda}+e_{\lambda} \cdot f \cdot e_{\lambda}\right)(x)\right| & =\left|f\left(\left(1-e_{\lambda}\right) x\left(1-e_{\lambda}\right)\right)\right| \\
& \leqq 2\left(\left\|a e_{\lambda}-a\right\|+\left\|e_{\lambda} a-a\right\|\right)\|x\|
\end{aligned}
$$

for each $f \in H$ and $x \in M(A)$. So for each $f \in H$

$$
\left\|f-\left(e_{\lambda} \cdot f+f \cdot e_{\lambda}-e_{\lambda} \cdot f \cdot e_{\lambda}\right)\right\| \leqq 2\left\|a e_{\lambda}-a\right\|+2\left\|e_{\lambda} a-a\right\|
$$

and therefore it is clear that (2) holds.

Now assume (2) holds and that $H$ is uniformly bounded by 1 . To prove that $H$ is $\beta$-equicontinuous, it will suffice to show that $H$ is contained in the polar of some basic neighborhood of 0 in $M(A)_{\beta}^{*}$. For each $\lambda \in \Lambda$ set $R_{\lambda} f=e_{\lambda} \cdot f+f \cdot e_{\lambda}-e_{\lambda} \cdot f \cdot e_{\lambda}$ for each $f \in M(A)_{\beta}^{*}$ and set $S_{\lambda} x=e_{\lambda} x+x e_{\lambda}-e_{\lambda} x e_{\lambda}$ for each $x \in M(A)_{\beta}$. Now choose a sequence $\left\{e_{\lambda_{n}}\right\}$ of elements from our approximate identity such that for each positive integer $n$ we have $\lambda_{n+1}>\lambda_{n},\left\|R_{\lambda_{n+1}} f-R_{\lambda_{n}} f\right\| \leqq 1 / 4^{n+1}$ for each $f \in H$, $\left\|e_{\lambda_{k}}-e_{\lambda_{k}} e_{\lambda_{n+1}}\right\| \leqq 1 / 9 \cdot 4^{n}$ for $k=1,2, \ldots, n$, and $\left\|e_{\lambda_{k}}-e_{\lambda_{n+1}} e_{\lambda_{k}}\right\|<1 / 9 \cdot 4^{n}$ for $k=1,2, \ldots, n$. Let $\left\{d_{k}\right\}$ be a sequence of elements in $A$ defined by $d_{5 k-4}=\left(3 / 2^{k+1}\right) e_{\lambda_{k}}$, $d_{5 k-3}=e_{\lambda_{k}}-e_{\lambda_{k}} e_{\lambda_{k+1}}, \quad d_{5 k-2}=e_{\lambda_{k}}-e_{\lambda_{k+1}} e_{\lambda_{k}}, \quad d_{5 k-1}=e_{\lambda_{k}}-e_{\lambda_{k}} e_{\lambda_{k+2}}, \quad$ and $d_{5 k}=e_{\lambda_{k}}$ $-e_{\lambda_{k+2}} e_{\lambda_{k}}$. It is clear that $d_{k} \rightarrow 0$ uniformly and $\left\|d_{k}\right\|<1$. Therefore, by Lemma 2.4, there exist sequences $\left\{b_{k}\right\}$ and $\left\{c_{k}\right\}$ of elements in $A$ and a hermitian element $a \in A,\|a\| \leqq 1$, such that $d_{k}=a b_{k}=c_{k} a$ and $\max \left\{\left\|b_{k}\right\|^{2},\left\|c_{k}\right\|^{2}\right\} \leqq\left\|d_{k}\right\|$. Set $a_{1}=8 a$. We now wish to show that $H \subset V_{a_{1}}^{0}$, where $V_{a_{1}}^{0}$ is the polar of

$$
V_{a_{1}}=\left\{x \in M(A):\left\|a_{1} x\right\| \leqq 1 \text { and }\left\|x a_{1}\right\| \leqq 1\right\}
$$

in $M(A)_{\beta}^{*}$. Since $d_{5 k-4}=a b_{5 k-4}=c_{5 k-4} a$, we have for each $x \in V_{a_{1}}$ that $\left\|x e_{\lambda_{k}}\right\|$ $=\left(2^{k+1} / 3\right)\left\|x a b_{5 k-4}\right\| \leqq 2^{k+1} / 3 \cdot 8$ and similarly $\left\|e_{\lambda_{k}} x\right\| \leqq 2^{k+1} / 3 \cdot 8$. It follows, by straightforward computations, that for each $f \in H$ and $x \in V_{a_{1}}$ that

$$
\left|R_{\lambda_{k}} f\left(x-S_{\lambda_{k+2}} x\right)\right| \leqq 1 / 2^{k+3}, \quad\left|R_{\lambda_{k+1}} f\left(x-S_{\lambda_{k+2}} x\right)\right| \leqq 1 / 2^{k+3},
$$

and

$$
\left\|S_{\lambda_{k}} x\right\| \leqq 2^{k+1} / 8 .
$$

These inequalities and the fact that $f=R_{\lambda_{1}} f+\sum_{k=1}^{\infty}\left(R_{\lambda_{k+1}} f-R_{\lambda_{k}} f\right)$ for each $f \in H$ imply that

$$
|f(x)| \leqq\left|f\left(S_{\lambda_{1}}(x)\right)\right|+\sum_{k=1}^{\infty}\left|\left(R_{\lambda_{k+1}} f-R_{\lambda_{k}} f\right)\left(x-S_{\lambda_{k+2}} x+S_{\lambda_{k+2}} x\right)\right|<1
$$

whenever $f \in H$ and $x \in V_{a_{1}}$. Hence $H \subset V_{a_{1}}^{0}$ and our proof is complete. 
We will now generalize a result due to L. LeCam [11, Proposition 2, p. 217] and J. R. Dorroh [8] that concerns the $\beta^{\prime}$ or bounded strict topology. The $\beta^{\prime}$ topology is the strongest locally convex topology for $M(A)$ that agrees with the $\beta$ topology on norm bounded sets. For a proof of existence, we refer the reader to [5] where an explicit neighborhood base is given. Another generalization of this theorem exists. F. D. Sentilles proved a similar result [15] in a Banach module setting and though we use the same technique his result does not seem to subsume our

Corollary 2.7. If $A$ is a $B^{*}$-algebra, then the $\beta$ and $\beta^{\prime}$ topologies for $M(A)$ give the same dual. Consequently, $\beta=\beta^{\prime}$.

Proof. By virtue of Theorem 2.1, the proof that the $\beta^{\prime}$ dual of $M(A)$ is $M(A)_{\beta}^{*}$ is similar to the one given for Corollary 2.2. Therefore, it remains to be shown that $\beta=\beta^{\prime}$. Let $W$ be an absolutely convex $\beta^{\prime}$-closed $\beta^{\prime}$-neighborhood of 0 . Then there exists a sequence $\left\{a_{n}\right\}$ of elements in $A$ such that $B_{n} \cap V_{a_{n}} \subset B_{n} \cap W$, where $V_{a_{n}}$ $=\left\{x \in M(A):\left\|a_{n} x\right\| \leqq 1\right.$ and $\left.\left\|x a_{n}\right\| \leqq 1\right\}$ and $B_{n}=\{x \in M(A):\|x\| \leqq n\}$. Set $D_{n}$ $=B_{n} \cap \dot{V}_{a_{n}}$ and $W^{\prime}$ equal the $\beta^{\prime}$-closed absolutely convex hull of $\bigcup D_{n}$. Then $W^{\prime} \subset W$, and $\left(W^{\prime}\right)^{0}=\bigcap\left(D_{n}\right)^{0}$, where $\left(W^{\prime}\right)^{0}$ and $\left(D_{n}\right)^{0}$ are the polars of $W^{\prime}$ and $D_{n}$ respectively in $M(A)_{\beta}^{*}$. We will show that $\left(W^{\prime}\right)^{0}$ is $\beta$-equicontinuous which implies that the $\beta$-closure of $W^{\prime}$ is a $\beta$-neighborhood. To this end, we will show that $e_{\lambda} \cdot f+f \cdot e_{\lambda}-e_{\lambda} \cdot f \cdot e_{\lambda} \rightarrow f$ uniformly on $\left(W^{\prime}\right)^{0}$, where $\left\{e_{\lambda}\right\}$ is an approximate identity for $A$ consisting of positive elements. Let $\varepsilon>0$. Choose a positive integer $n$ so that $1 / n<\varepsilon$ and then choose a $\lambda_{0}$ so that for $\lambda \leqq \lambda_{0},\left\|\left(1-e_{\lambda}\right) a_{n}\right\|<1 / n$ and $\left\|a_{n}\left(1-e_{\lambda}\right)\right\|$ $<1 / n$. Hence $\left\{n\left(1-e_{\lambda}\right) x\left(1-e_{\lambda}\right): x \in B_{1}\right\} \subset D_{n}$ for $\lambda \geqq \lambda_{0}$. Therefore for $f \in\left(W^{\prime}\right)^{0}$, $x \in B_{1}$, and $\lambda \geqq \lambda_{0}$

$$
\left|\left(f-e_{\lambda} \cdot f-f \cdot e_{\lambda}+e_{\lambda} \cdot f \cdot e_{\lambda}\right)(x)\right|=\left|f\left(\left(1-e_{\lambda}\right) x\left(1-e_{\lambda}\right)\right)\right|<1 / n<\varepsilon .
$$

In other words, $\left\|f-e_{\lambda} \cdot f-f \cdot e_{\lambda}+e_{\lambda} \cdot f \cdot e_{\lambda}\right\|<\varepsilon$ for all $f \in\left(W^{\prime}\right)^{0}$ and $\lambda \geqq \lambda_{0}$. Thus, by Theorem 2.6, $\left(W^{\prime}\right)^{0}$ is a $\beta$-equicontinuous and our proof is complete.

It is well known that the bidual $A^{* *}$ of a $B^{*}$-algebra $A$ is a $W^{*}$-algebra, and when $A$ is canonically imbedded into $A^{* *}, A$ is a *-subalgebra of $A^{* *}$. We will now consider the case when $M(A)$ is isometrically *-isomorphic to $A^{* *}$. For example, if $A$ is also an annihilator algebra, then this is true.

COROllary 2.8. Let $A$ be a $B^{*}$-algebra such that $M(A)$ is isometrically *-isomorphic to $A^{* *}$. Then $M(A)_{B}$ is a Mackey space.

Proof. The proof follows from Corollary 2.2, Corollary 2.3, Corollary 2.7, and [1, Theorem II.7, p. 292].

\section{Proof of Theorem I and Theorem II.}

Lemma 3.1. Let $\left\{A_{\lambda}: \lambda \in \Lambda\right\}$ be a family of $B^{*}$-algebras and let $A=\left(\sum A_{\lambda}\right)_{0}$. Then $M(A)$ is isometrically *-isomorphic to $\sum M\left(A_{\lambda}\right)$. 
Proof. Let $(R, S) \in M(A)$ and let $\lambda \in \Lambda$. Define $R_{\lambda}$ and $S_{\lambda}$ on $A_{\lambda}$ by the formula $R_{\lambda}(a(\lambda))=(R(a))(\lambda)$ and $S_{\lambda}(a(\lambda))=(S(a))(\lambda)$ for each $a \in A$. To see that $R_{\lambda}$ and $S_{\lambda}$ are well defined, observe that if $a \in A$, with $a(\lambda)=0$, and if $\left\{e_{\alpha}\right\}$ is an approximate identity for $A$, then by [4, Proposition 2.5, p. 80$]$,

$$
R(a)(\lambda)=\lim R\left(e_{\alpha} a\right)(\lambda)=\lim R\left(e_{\alpha}\right)(\lambda) a(\lambda)=0,
$$

and similarly, $S(a)(\lambda)=0$. It is straightforward to show that $\left(R_{\lambda}, S_{\lambda}\right) \in M\left(A_{\lambda}\right)$ and that $\left\|\left(R_{\lambda}, S_{\lambda}\right)\right\| \leqq\|(R, S)\|$, so define the map $\mu: M(A) \rightarrow \sum M\left(A_{\lambda}\right)$ by the formula $\mu((R, S))(\lambda)=\left(R_{\lambda}, S_{\lambda}\right)$. It is clear that $\mu$ is a $*$-isomorphism from $M(A)$ into $\sum M\left(A_{\lambda}\right)$ and that $\|\mu((R, S))\| \leqq\|(R, S)\|$ for all $(R, S) \in M(A)$. Now for $(R, S) \in M(A)$ and $a \in A,\|a\| \leqq 1$,

$$
\begin{aligned}
\|R(a)\| & =\sup \{\|R(a)(\lambda)\|: \lambda \in \Lambda\}=\sup \left\{\left\|R_{\lambda}(a(\lambda))\right\|: \lambda \in \Lambda\right\} \\
& \leqq \sup \left\{\left\|R_{\lambda}\right\|: \lambda \in \Lambda\right\}=\sup \left\{\left\|\left(R_{\lambda}, S_{\lambda}\right)\right\|: \lambda \in \Lambda\right\}=\|\mu((R, S))\| .
\end{aligned}
$$

In other words, $\|(R, S)\|=\|\mu((R, S))\|$. Therefore to complete the proof we need to show that $\mu$ is onto. Let $\sum\left(R_{\lambda}, S_{\lambda}\right) \in \sum M\left(A_{\lambda}\right)$ and define $(R(a))(\lambda)=$ $R_{\lambda}(a(\lambda))$ and $(S(a))(\lambda)=S_{\lambda}(a(\lambda))$ for each $a \in A$ and $\lambda \in \Lambda$. But it is clear that $(R, S) \in M(A)$ and $\mu((R, S))=\sum\left(R_{\lambda}, S_{\lambda}\right)$. Hence $\mu$ is onto and our proof is complete.

Lemma 3.2. Let $\left\{A_{\lambda}: \lambda \in \Lambda\right\}$ be a family of $B^{*}$-algebras. Then the following statements are eqivalent:

(1) If $A=\left(\sum_{\lambda \in \Lambda} A_{\lambda}\right)_{0}$, then $M(A)_{\beta}$ is a Mackey space.

(2) If $\Lambda_{0}$ is a countable subset of $\Lambda$ and $A_{0}=\left(\sum_{\lambda \in \Lambda_{0}} A_{\lambda}\right)_{0}$, then $M\left(A_{0}\right)_{\beta}$ is a Mackey space.

Proof. By virtue of Theorem 2.6, Lemma 3.1, and [10, p. 173], it is easy to show that (1) implies (2). Now let $H$ be a $\beta$-weak* compact convex circled subset of $M(A)_{\beta}^{*}$ and let $\phi_{\lambda}$ denote the restriction map from $M(A)$ onto $M\left(A_{\lambda}\right)$, where $M\left(A_{\lambda}\right)$ is now viewed as a subspace of $M(A)$. Set $\Lambda_{0}=\left\{\lambda \in \Lambda:\left\|\phi_{\lambda} f\right\|>0\right.$ for some $f \in H\}$. If $\Lambda_{0}$ is countable, then (2), together with Theorem 2.6, Lemma 3.1, and [10, p. 173], implies that $H$ is $\beta$-equicontinuous and therefore, by [10, p. 173], (2) implies (1). Hence, it remains to be shown that $\Lambda_{0}$ is countable.

For each $\lambda \in \Lambda_{0}$ choose an $x_{\lambda} \in M\left(A_{\lambda}\right),\left\|x_{\lambda}\right\| \leqq 1$, so that for some $f \in H$ we have $f\left(x_{\lambda}\right) \neq 0$. Now define $x \in M(A)$ by the formula

$$
\begin{aligned}
x(\lambda) & =x_{\lambda} & & \text { if } \lambda \in \Lambda_{0}, \\
& =0 & & \text { if } \lambda \notin \Lambda_{0},
\end{aligned}
$$

where we now view $M(A)$ as $\sum_{\lambda \in \Lambda} M\left(A_{\lambda}\right)$, and then define the map

$$
T: C(\Lambda)_{\beta} \rightarrow M(A)_{\beta}
$$

by the formula $T(\alpha)(\lambda)=\alpha(\lambda) x(\lambda)$ for each $\alpha \in C(\Lambda)$ and $\lambda \in \Lambda$. Here the topology 
for $\Lambda$ is the discrete topology. Let $\left\{\alpha_{i}\right\}$ be a norm bounded net in $C(\Lambda)$ that converges to zero in the strict topology. It is straightforward to show that the net $\left\{T\left(\alpha_{i}\right)\right\}$ in $M(A)$ converges to zero in the strict topology and therefore, by virtue of Corollary 2.7, $T$ is $\beta$-continuous. This implies that $T$ has a well-defined adjoint map $T^{*}: M(A)_{B}^{*} \rightarrow C(\Lambda)_{B}^{*}$, which is continuous when both range and domain have their $\beta$-weak* topologies. It follows that $T^{*}(H)$ is $\beta$-weak* compact and therefore, by virtue of $\left[6\right.$, Theorem 2.6, p. 478] and [6, Theorem 2.2, p. 476], $\Lambda_{0}$ is countable. Hence our proof is complete.

Lemma 3.3. Let $A$ be $a B^{*}$-algebra and let $a$ and $b$ be hermitian elements in $A$ such that $1 \geqq\|a\| \geqq\|b\|$. Then $\|a+b\| \leqq 1+2\|a b\|$.

Proof. Let $\sigma$ be the smallest number such that

$$
\|c+d\| \leqq 1+\sigma
$$

for all hermitian elements $c$ and $d$ in $A$, where $1 \geqq\|c\| \geqq\|d\|$ and $\|c d\| \leqq\|a b\|$. It is clear that such a number exists. Now if $\sigma>2\|a b\|$, then $\|c+d\|^{2}=\left\|(c+d)^{2}\right\|$ $\leqq\left\|c^{2}+d^{2}\right\|+2\|c d\| \leqq 1+\sigma+2\|a b\|<(1+\sigma)^{2}$ for all hermitian elements $c$ and $d$ in $A$, where $1 \geqq\|c\| \geqq\|d\|$ and $\|c d\| \leqq\|a b\|$. But this contradicts (3.1), so $\sigma \leqq 2\|a b\|$ and our proof is complete.

The author would like to thank Professor L. Eifler for the suggestion of the argument given for Lemma 3.3. This argument eliminated a longer proof.

REMARK. It follows immediately from Lemma 3.3 that for each pair of hermitian elements $a, b$ in a $B^{*}$-algebra $A$ the inequality $\|a+b\| \leqq\|a\|+2\|a b\| /\|a\|$ holds whenever $\|a\| \geqq\|b\|$ and $\|a\| \neq 0$. In fact, there is a smallest number $k$ such that $\|a+b\| \leqq\|a\|+k\|a b\| /\|a\|$ and this, in a sense, is a generalization of the triangle inequality for $B^{*}$-algebras. But $k=1$ when the $B^{*}$-algebra $A$ is commutative, and this fact suggests the following question: Is it true that $k=1$ only if $A$ is commutative?

Proof of Theorem I. Let $\left\{A_{k}\right\}_{k=1}^{\infty}$ be a sequence of $B^{*}$-algebras such that $M\left(A_{k}\right)_{\beta}$ is a Mackey space for each positive integer $k$. If we show that $M(A)_{\beta}$ is a Mackey space, where $A=\left(\sum_{k=1}^{\infty} A_{k}\right)_{0}$, then by virtue of Lemma 3.2 the proof will be complete. To this end, it will suffice to show that each $\beta$-weak* compact circled convex subset of $M(A)_{\beta}^{*}$ is $\beta$-equicontinuous. Now suppose that $H$ is a $\beta$-weak* compact circled convex subset of $M(A)_{\beta}^{*}$ that is not $\beta$-equicontinuous. Since $H$ is $\beta$-weak* compact, $H$ is uniformly bounded and we can assume, without loss of generality, that $H$ is uniformly bounded by 1 . Let $\left\{e_{\delta}: \delta \in \Delta\right\}$ be an approximate identity for $A$ consisting of positive elements. Then by virtue of Theorem 2.6 there exists an $\varepsilon>0$ such that for each $\delta_{0} \in \Delta$ we have

$$
\left\|f-e_{\delta} \cdot f-f \cdot e_{\delta}+e_{\delta} \cdot f \cdot e_{\delta}\right\| \geqq 4 \varepsilon
$$

for some $f \in H$ and $\delta>\delta_{0}$. We will now define by induction a sequence of triples $\left\{\left(f_{k}, x_{k}, n_{k}\right)\right\}_{k=1}^{\infty}$ that satisfies the following conditions:

(1) $f_{k} \in H, x_{k} \in M(A)$, and $n_{k}$ is a positive integer less than $n_{k+1}$. 
(2) $\left\|x_{k}\right\| \leqq 1, x_{k}(q)=0$ for each positive integer $q \leqq n_{k-1}$ or $q>n_{k}$, where $M(A)$ is now viewed as $\sum_{k=1}^{\infty} M\left(A_{k}\right)$.

(3) $\left|f_{k}\left(x_{k}\right)\right| \geqq \varepsilon$.

By virtue of (3.2) there exists an $f_{1}$ in $H$, a $\delta$ in $\Delta$, and a $y$ in the unit ball of $M(A)$ such that $\left|f_{1}\left(\left(1-e_{\delta}\right) y\left(1-e_{\delta}\right)\right)\right| \geqq 3 \varepsilon$. Since $M(A)_{\beta}^{*}$ under the strong topology is isometrically isomorphic to $A^{*}$ and $A^{*}$ is isometrically isomorphic to the $L^{1}$ direct sum of $\left\{A_{k}^{*}\right\}_{k=1}^{\infty}$, we can find a positive integer $n_{1}$ such that $\left|f_{1}\left(x_{1}\right)\right| \geqq \varepsilon$, where $x_{1}$ is the element in $M(A)$ defined by $x_{1}(q)=\left(\left(1-e_{\delta}\right) y\left(1-e_{\delta}\right)\right)(q)$ for $q=1,2, \ldots, n_{1}$ and $x_{1}(q)=0$ for $q>n_{1}$. It is clear that $\left(f_{1}, x_{1}, n_{1}\right)$ satisfies conditions (1), (2), and (3). Now assume that $\left(f_{k}, x_{k}, n_{k}\right)$ has been defined for $k=1,2, \ldots, p$. Let $B_{n_{p}}$ be the subspace of $A$ defined by $B_{n_{p}}=\sum_{k=1}^{n_{p}} A_{k}$ and let $\phi$ denote the restriction mapping from $M(A)$ to $M\left(B_{n_{p}}\right)=\sum_{k=1}^{n_{p}} M\left(A_{k}\right)$. It is straightforward to show, by using Theorem 2.6, that $M\left(B_{n_{p}}\right)_{\beta}$ is a Mackey space and therefore, by virtue of Theorem 2.6, [10, p. 173], and (3.2), there exists an $f_{p+1}$ in $H$, a $\delta$ in $\Delta$, and a $y$ in the unit ball of $M(A)$ such that $\left|f_{p+1}\left(\left(1-e_{\delta}\right) y\left(1-e_{\delta}\right)\right)\right| \geqq 3 \varepsilon$ and

$$
\left\|\phi\left(f_{p+1}-e_{\delta} \cdot f_{p+1}-f_{p+1} \cdot e_{\delta}+e_{\delta} \cdot f_{p+1} \cdot e_{\delta}\right)\right\|<\varepsilon .
$$

By virtue of (3.3) and the fact that $M(A)_{\beta}^{*}$ under the strong topology is isometrically isomorphic to the $L^{1}$ direct sum of $\left\{A_{k}^{*}\right\}_{k=1}^{\infty}$, we can find a positive integer $n_{p+1}>n_{p}$ such that $\left|f\left(x_{p+1}\right)\right| \geqq \varepsilon$, where $x_{p+1}$ is the element in $M(A)$ defined by $x_{p+1}(q)$ $=\left(\left(1-e_{\delta}\right) y\left(1-e_{\delta}\right)\right)(q)$ for $n_{p}<q \leqq n_{p+1}$ and $x_{p+1}(q)=0$ otherwise. It is clear that $\left(f_{p+1}, x_{p+1}, n_{p+1}\right)$ satisfies conditions (1), (2), and (3), and our induction is complete. Now let $x$ be the element in $M(A)$ defined by $x(q)=x_{k}(q)$ when $n_{k-1}<q \leqq n_{k}$. Then define the map $T:\left(l^{\infty}, \beta\right) \rightarrow M(A)_{\beta}$ by the formula $T(\alpha)(q)=\alpha(q) x(q)$ for each $\alpha \in l^{\infty}$ and positive integer $q$. By virtue of Corollary 2.7, it is straightforward to show that $T$ is continuous. Hence $T$ has a well-defined adjoint map $T^{*}: M(A)_{\beta}^{*}$ $\rightarrow l^{1}$, which is continuous when both range and domain have the $\beta$-weak* topologies. Thus, $T^{*}(H)$ is a $\beta$-weak* compact subset of $l^{1}$ and this implies, by virtue of [6, Theorem 2.4, p. 477], that $T^{*}(H)$ is $\beta$-equicontinuous in $l^{1}$. Since $\sum_{k=1}^{q} \alpha(k) x(k)$ converges in the strict topology to $T(\alpha)$ as $q \rightarrow \infty$ for each $\alpha \in l^{\infty}$, we see that $T^{*} f(\alpha)=f(T(\alpha))=\sum_{k=1}^{\infty} \alpha(k) f(x(k))$ for each $f \in M(A)_{\beta}^{*}$ and $\alpha \in l^{\infty}$. So $T^{*} f=$ $\{f(x(k))\}_{k=1}^{\infty}$. Since $T^{*}(H)$ is $\beta$-equicontinuous, there exists, by virtue of [6, Theorem 2.2, p. 476], a positive integer $N$ such that $\sum_{k=N+1}^{\infty}|f(x(k))|<\varepsilon$ for each $f \in H$. This implies that $\left|f\left(x_{q}\right)\right| \leqq \sum_{k=n_{q}}^{n_{q}+1}|f(x(k))|<\varepsilon$ for $n_{q}>N$. This holds for all $f \in H$ and in particular $\left|f_{q}\left(x_{q}\right)\right|<\varepsilon$. But this contradicts (3). Hence $H$ is $\beta$-equicontinuous and our proof is complete.

Proof of Theorem II. Let $A$ be a $B^{*}$-algebra. If condition (1) holds, then it follows from Corollary 2.8 that $M(A)_{\beta}$ is a Mackey space. Now assume that $A$ has a countable approximate identity. To show that $M(A)_{\beta}$ is a Mackey space it will suffice to show that every $\beta$-weak* compact subset of $M(A)_{\beta}^{*}$ is $\beta$-equicontinuous [10, p. 173]. Suppose that $H$ is a $\beta$-weak* compact subset of $M(A)_{\beta}^{*}$ that is not $\beta$-equicontinuous. Since $H$ is $\beta$-weak* compact, $H$ is uniformly bounded, and 
without loss of generality we can assume that $H$ is uniformly bounded by 1 . Suppose $\left\{d_{k}\right\}_{k=1}^{\infty}$ is an approximate identity for $A$ consisting of positive elements. We may assume that for each positive integer $n$

$$
\left\|d_{n+1} d_{k}-d_{k}\right\|<1 / n \cdot 2^{n+3}
$$

for $k=1,2, \ldots, n$. Now because of Theorem 2.6 there exists an $\varepsilon>0$ such that for each positive integer $N$ the inequality

$$
\left\|f-d_{n} \cdot f-f \cdot d_{n}+d_{n} \cdot f \cdot d_{n}\right\| \geqq 5 \varepsilon
$$

holds for some $f \in H$ and integer $n>N$. We will now define by induction a sequence of quadruples $\left\{\left(f_{k}, a_{k}, n_{2 k-1}, n_{2 k}\right)\right\}_{k=1}^{\infty}$ that satisfies the following conditions:

(a) $f_{k} \in H, a_{k}$ is a hermitian element in the unit ball of $A$, and $n_{2 k-1}, n_{2 k}$ are positive integers such that $n_{2 k-1}<n_{2 k}<n_{2 k+1}$.

(b) $\left|f_{k}\left(d_{n_{2 k}}\left(1-d_{n_{2 k-1}}\right) a_{k}\left(1-d_{n_{2 k-1}}\right) d_{n_{2 k}}\right)\right| \geqq \varepsilon$.

By virtue of (3.5) and Corollary 2.3, it is straightforward to show that there exist an $f_{1} \in H$, a hermitian element $a_{1}$ in the unit ball of $A$, and positive integers $n_{1}, n_{2}$ with $n_{1}<n_{2}$ such that

$$
\left|f_{1}\left(d_{n_{2}}\left(1-d_{n_{1}}\right) a_{1}\left(1-d_{n_{1}}\right) d_{n_{2}}\right)\right| \geqq \varepsilon .
$$

Thus $\left(f_{1}, a_{1}, n_{1}, n_{2}\right)$ satisfies (a) and (b). Now suppose the quadruple $\left(f_{k}, a_{k}\right.$, $n_{2 k-1}, n_{2 k}$ ) has been defined for $k=1,2, \ldots, p$ so that conditions (a) and (b) have been satisfied. Again, by virtue of (3.5) and Corollary 2.3, it is straightforward to show that there exist an $f_{p+1} \in H$, a hermitian element $a_{p+1}$ in the unit ball of $A$, and positive integers $n_{2 p+1}, n_{2 p+2}$ with $n_{2 p}<n_{2 p+1}<n_{2 p+2}$ such that

$$
\left|f_{p+1}\left(d_{n_{2 p+1}}\left(1-d_{n_{2 p+1}}\right) a_{p+1}\left(1-d_{n_{2 p+1}}\right) d_{n_{2 p+2}}\right)\right| \geqq \varepsilon
$$

and our induction is complete. Set $x_{k}=d_{n_{2 k}}\left(1-d_{n_{2 k-1}}\right) a_{k}\left(1-d_{n_{2 k-1}}\right) d_{n_{2 k}}$ and $e_{k}=d_{2 k}$. Because of (3.4), (a), and (b), $\left\{\left(f_{k}, x_{k}, e_{k}\right)\right\}_{k=1}^{\infty}$ is a sequence of triples such that the following conditions hold:

(a) $f_{k} \in H, x_{n}$ is an hermitian element in the unit ball of $A$, and $e_{k} \in A$.

(b) $\left\{e_{k}\right\}$ is an approximate identity for $A$ consisting of positive elements.

(c)' For each positive integer $p,\left\|e_{p} x_{k}\right\|=\left\|x_{k} e_{p}\right\|<1 / 2^{k}$ for $k=p+1, p+2, \ldots$ and $\left\|x_{p+1} x_{k}\right\|=\left\|x_{k} x_{p+1}\right\|<1 / p \cdot 2^{p+2}$ for $k=1,2, \ldots, p$.

$(\mathrm{d})^{\prime}\left|f_{k}\left(x_{k}\right)\right| \geqq \varepsilon$.

Let $\alpha=\left\{\alpha_{k}\right\}_{k=1}^{\infty}$ belong to $l^{\infty}$. By virtue of Lemma 3.3, it is straightforward to show that $\left\|\sum_{k=1}^{n} \alpha_{k} x_{k}\right\| \leqq\|\alpha\|_{\infty} \sum_{k=1}^{n} 1 / 2^{k-1} \leqq 2\|\alpha\|_{\infty}$ for each positive integer $n$. This inequality and the fact that $\left\|e_{n} x_{p}\right\|=\left\|x_{p} e_{n}\right\|<1 / 2^{p}$ for $p \geqq n+1$, imply that the sequence of partial sums $\left\{\sum_{k=1}^{n} \alpha_{k} x_{k}\right\}_{n=1}^{\infty}$ is $\beta$-Cauchy. Since $M(A)_{\beta}$ is complete [4, Proposition 3.6, p. 83], we may define the map $T:\left(l^{\infty}, \beta\right) \rightarrow M(A)_{\beta}$ by the formula $T(\alpha)=\sum_{k=1}^{\infty} \alpha_{k} x_{k}$, where $\alpha=\left\{\alpha_{k}\right\}_{k=1}^{\infty}$ and $\sum_{k=1}^{\infty} \alpha_{k} x_{k}$ is the $\beta$-limit of the partial sums. By virtue of Corollary 2.7, it is straightforward to show that $T$ is continuous and therefore $T$ has a well-defined adjoint map $T^{*}: M(A)_{\beta}^{*} \rightarrow l^{1}$, 
which is continuous when both range and domain have the $\beta$-weak* topologies. Thus $T^{*}(H)$ is a $\beta$-weak* compact subset of $l^{1}$ and this implies, by virtue of [6, Theorem 2.4, p. 477], $T^{*}(H)$ is $\beta$-equicontinuous in $l^{1}$. Observe that $T^{*} f(\alpha)$ $=f(T(\alpha))=\sum_{k=1}^{\infty} \alpha_{k} f\left(x_{k}\right)$ for each $\alpha \in l^{\infty}$ and $f \in M(A)_{\beta}^{*}$, so that $T^{*} f=\left\{f\left(x_{k}\right)\right\}_{k=1}^{\infty}$. Since $T^{*}(H)$ is $\beta$-equicontinuous, there exists by virtue of [6, Theorem 2.2, p. 476] a positive integer $N$ such that $\sum_{k=N+1}^{\infty}\left|f\left(x_{k}\right)\right|<\varepsilon$ for each $f \in H$. Thus, for $f \in H$ and $k>N$ we have $\left|f\left(x_{k}\right)\right|<\varepsilon$ and in particular $\left|f_{k}\left(x_{k}\right)\right|<\varepsilon$ for $k>N$. But this contradicts (d)'. Hence $H$ is $\beta$-equicontinuous and our proof is complete.

\section{REFERENCES}

1. C. A. Akemann, The dual space of an operator algebra, Trans. Amer. Math. Soc. 126 (1967), 286-302. MR 34 \#6549.

2. N. Bourbaki, Livre III. Topologie générale, Actualités Sci. Indust., no. 1196, Hermann, Paris, 1953. MR 14, 1106.

3. R. C. Buck, Bounded continuous functions on a locally compact space, Michigan Math. J. 5 (1958), 95-104. MR 21 \#4350.

4. R. C. Busby, Double centralizers and extensions of $C^{*}$-algebras, Trans. Amer. Math. Soc. 132 (1968), 79-99. MR 37 \#770.

5. H. S. Collins, Completeness and compactness in linear topological spaces, Trans. Amer. Math. Soc. 79 (1955), 256-280. MR 16, 1030.

6. J. B. Conway, The strict topology and compactness in the space of measures. II, Trans. Amer. Math. Soc. 126 (1967), 474-486. MR 34 \#6503.

7. J. Dixmier, Les $C^{*}$-algèbres et leurs représentations, Cahiers Scientifiques, fasc. 29, Gauthier-Villars, Paris, 1964. MR 30 \#1404.

8. J. R. Dorroh, The localization of the strict topology via bounded sets, Proc. Amer. Math. Soc. 20 (1969), 413-414. MR 38 \#3721.

9. E. Hewitt, The ranges of certain convolution operators, Math. Scand. 15 (1964), 147-155. MR 32 \#4471.

10. J. L. Kelley and I. Namioka, Linear topological spaces, The University Series in Higher Math., Van Nostrand, Princeton, N. J., 1963. MR 29 \#3851.

11. L. LeCam, Convergence in distribution of stochastic processes, Univ. Calif. Publ. Statist. 2 (1957), 207-236. MR 19, 128.

12. C. Rickart, General theory of Banach algebras, The University Series in Higher Math., Van Nostrand, Princeton, N. J., 1960. MR 22 \#5903.

13. A. P. Robertson and W. J. Robertson, Topological vector spaces, Cambridge Univ. Press, New York, 1964. MR 28 \#5318.

14. S. Sakai, On topological properties of $W^{*}$-algebras, Proc. Japan Acad. 33 (1957), 439-444. MR 20 \#5437.

15. F. D. Sentilles, Conditions for equality of the Mackey and strict topologies, Bull. Amer. Math. Soc. 76 (1970), 107-112.

16. F. D. Sentilles and D. C. Taylor, Factorization in Banach algebras and the general strict topology, Trans. Amer. Math. Soc. 142 (1969), 141-152.

17. Ju-kwei Wang, Multipliers of commutative Banach algebras, Pacific J. Math. 11 (1961), 1131-1149. MR 25 \#1462.

UNIVERSITY OF MISSOURI, Columbia, Missouri 65201 